\title{
Capillary Chromatography Based on Tube Radial Distribution of Aqueous-Organic Mixture Carrier Solvents: Introduction of Double Tubes Having Different Inner Diameters to the System
}

\author{
Koju YAMAdA, Naoya Jinno, Masahiko HASHIMOTo, and Kazuhiko TsuKagoSHI ${ }^{\dagger}$ \\ Department of Chemical Engineering and Materials Science, Faculty of Science and Engineering, \\ Doshisha University, Kyotanabe, Kyoto 610-0321, Japan
}

\begin{abstract}
A tube radial distribution chromatography (TRDC) system was previously developed using an open capillary tube and an aqueous-organic solvent (water-acetonitrile-ethyl acetate) mixture as a carrier solution. In this study, we introduced double capillary tubes having different inner diameters to the system. The tubes were fused-silica capillary tubes with 100 and $250 \mu \mathrm{m}$ i.d.; the smaller tube was inserted into the larger one through a T-type joint. Water-acetonitrile mixture (volume ratio 3:1) and acetonitrile-ethyl acetate mixture (volume ratio 4:1) solutions were delivered into the large tube from the inside through the small tube and from the outside through the joint, respectively, and then mixed through the large tube to provide a water-acetonitrile-ethyl acetate carrier solution. The carrier solution was further fed into the large tube and then to an absorption detector. By changing the flow rates of the mixture solutions in both tubes, we could control the component ratio of carrier solvents in the carrier solution, yielding either organic solvent-rich, water-acetonitrile-ethyl acetate (volume ratio 3:33:8) or water-rich, water-acetonitrile-ethyl acetate (volume ratio 24:12:1). A model analyte-mixture solution of 1-naphthol and 2,6-naphthalenedisulfonic acid was eluted in this order with the organic solvent-rich carrier solution and eluted in the reverse order with the water-rich carrier solution. We discussed the chromatographic data together with the analytical conditions from the viewpoint of the tube radial distribution of the carrier solvents.
\end{abstract}

(Received November 28, 2009; Accepted February 11, 2010; Published April 10, 2010)

\section{Introduction}

Commercially available capillary tubes, e.g., fused-silica tubes, have uniform narrow bores, inert inner surfaces, and good flexibility, and are convenient for use with various analytical instruments. Among analytical techniques that make use of these tubes, various types of capillary chromatography, including capillary electrochromatography ${ }^{1,2}$ and capillary high-performance liquid chromatography using packed and monolithic capillary columns, ${ }^{3,4}$ have attracted considerable attention for use in the fields of analytical chemistry and separation science. Capillary chromatography using open fused-silica capillary tubes has also been investigated and the results indicated interesting and unique characteristics. ${ }^{5-8}$ Most capillary chromatography systems feature rapid measurements, easy procedures, inexpensive and small apparatus, small sample volumes, and low costs.

We reported a capillary chromatography system using open capillary tubes made of either fused silica, polyethylene, or poly(tetrafluoroethylene), and a water-hydrophilic-hydrophobic organic solvent mixture carrier solution.9,10 Various analyte-mixture solutions of hydrophilic and hydrophobic molecules, such as 2,6-naphthalenedisulfonic acid and 1-naphthol, Eosin Y and perylene, bis[ $N, N$-bis(carboxymethyl)-

† To whom correspondence should be addressed.

E-mail: ktsukago@mail.doshisha.ac.jp aminomethyl]fluorescein and 1,1'-bi-2-naphthol, and 2,7-naphthalenedisulfonic acid and p-nitroaniline, were examined in the chromatography system. We call this a tube radial distribution chromatography (TRDC) system. Separation in the TRDC system is performed without the use of any specific packed or monolithic capillary tubes, gel additives, surfactants, salts, or high-voltage devices. To date, in the TRDC system, aqueous-organic solvent carrier solutions were prepared with a water-acetonitrile-ethyl acetate mixture at various volume ratios to become homogeneous solutions, ${ }^{9,10}$ and were then delivered into a single capillary tube by a microsyringe pump. To change the component ratio of solvents in the carrier solution, we had to prepare the solution from the beginning and introduce it again into the TRDC system.

As investigation of the TRDC system has just begun, all investigations regarding apparatus, analytical procedures, conditions, subjects, separation mechanism, and applications must await future reports. In this study, we introduced double capillary tubes having different inner diameters to the TRDC system, instead of the single capillary tube just mentioned above. The double tubes consisted of a small- and a large-inner diameter fused-silica capillary tube; the small tube was inserted into the large tube through a T-type joint. Water-acetonitrile mixture and acetonitrile-ethyl acetate mixture solutions were delivered into the large tube from the inside and outside, respectively, and then mixed through the large tube to provide a water-acetonitrile-ethyl acetate carrier solution. By controlling the flow rates of the mixture solutions in the two tubes, 


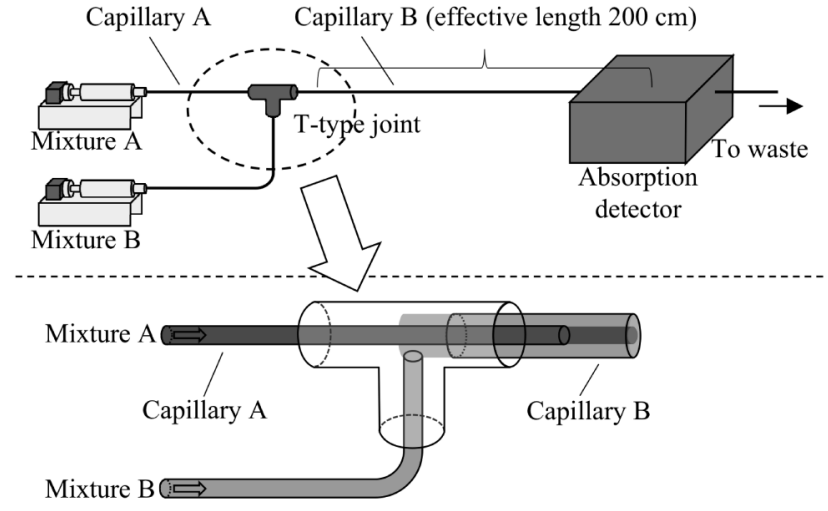

Fig. 1 Schematic of the present TRDC system equipped with double capillary tubes.

we could change the component ratio of a water-acetonitrile-ethyl acetate carrier solution, resulting in an organic solvent-rich solution and a water-rich carrier solution. We were thus able to separate and detect a model analyte-mixture solution of 1-naphthol and 2,6-naphthalenedisulfonic acid. Separation performance in the TRDC system was discussed based on the obtained chromatograms and the data concerning analytical conditions.

\section{Experimental}

\section{Chemicals and materials}

Water was purified using a water purification system (Elix UV 3; Millipore Co.). All reagents were commercially available and of analytical grade. 1-Naphthol, 2,6-naphthalenedisulfonic acid, acetonitrile, and ethyl acetate were purchased from Wako Pure Chemical Industries, Ltd. Fused-silica capillary tubes $(100 \mu \mathrm{m}$ i.d. and $200 \mu \mathrm{m}$ o.d. as well as $250 \mu \mathrm{m}$ i.d. and $350 \mu \mathrm{m}$ o.d.) were purchased from GL Science.

\section{Apparatus and analytical procedures}

Figure 1 shows a schematic diagram of the TRDC system comprising double capillary tubes, two microsyringe pumps (MF-9090; Bioanalytical Systems, Inc.), and an absorption detector (modified SPD-10AV spectrophotometric detector; Shimadzu Co.); an enlarged image of the tubes and T-type joint is also shown (though the thickness of the tubes is not accurately expressed in the enlarged image). The tubes were fused-silica capillary tubes of 100 and $250 \mu \mathrm{m}$ i.d. (called Capillaries A and $\mathrm{B}$, respectively); Capillary A was inserted into Capillary B through a T-type joint. Water-acetonitrile mixture (volume ratio 3:1) and acetonitrile-ethyl acetate mixture (volume ratio 4:1) solutions (called Mixtures A and B, respectively) were delivered into Capillary B from the inside through Capillary A and from the outside through the joint, respectively, with the corresponding microsyringe pumps, then mixed through Capillary B. The resulting water-acetonitrile-ethyl acetate carrier solution in Capillary B was delivered to an adsorption detector; the distance from the outlet of Capillary A to the detector (the effective capillary length for separation) was 100 or $200 \mathrm{~cm}$. The temperature of Capillary B having a 200-cm effective length was adjusted by dipping a part of the capillary tube $(\mathrm{ca} .130 \mathrm{~cm})$ in water maintained at a definite temperature in a vessel with stirring.
By changing the flow rates of the mixture solutions (Mixtures $\mathrm{A}$ and $\mathrm{B}$ ), we could control the component ratio of carrier solvents in the carrier solution that was fed into Capillary B. For example, an organic solvent-rich carrier solution (wateracetonitrile-ethyl acetate, volume ratio 3:33:8) was delivered at flow rates of 1.0 and $8.0 \mu \mathrm{min}^{-1}$ for Mixtures A and B, respectively. A water-rich carrier solution (water-acetonitrileethyl acetate, volume ratio 24:12:1) was delivered at flow rates of 8.0 and $1.0 \mu \mathrm{min}^{-1}$ for Mixtures A and B, respectively.

An analyte-mixture solution of 1-naphthol and 2,6-naphthalenedisulfonic acid was prepared with Mixture A and introduced directly into the capillary inlet of Capillary A for $15 \mathrm{~s}$ from a height of $20 \mathrm{~cm}$ by the gravity method. After analyte injection, the capillary inlet was connected to a microsyringe set on the microsyringe pump. Mixtures A and B were fed at definite flow rates, respectively, to yield the desired organic solvent-rich or water-rich carrier solution in Capillary B. On-capillary absorption detection $(254 \mathrm{~nm})$ was performed with the detector.

\section{Results and Discussion}

\section{Separation in the TRDC system}

Separation in the TRDC system was proposed in our previous paper, ${ }^{10}$ briefly repeated below. Water and organic solvent in the carrier solution do not disperse uniformly in the capillary, leading to the generation of a water-rich phase and an organic solvent-rich phase in the tube. A major solvent phase forms around the middle of the tube far from the inner wall as an inner phase, while a minor solvent phase is generated near the inner wall as an outer or capillary wall phase; when using a water-rich carrier solution, a major solvent phase or a water-rich phase forms around the middle of the tube as an inner phase, while when using an organic solvent-rich carrier solution, a major solvent phase or an organic solvent-rich phase forms as an inner phase. The tube radial distribution of the solvent molecules in the carrier solution is thus caused by the flow in the capillary tube under laminar flow conditions. Subsequently, the analytes are delivered through the tube, being distributed between the inner and outer (capillary wall) phases. The analyte little partitioned in the outer phase near the inner wall of the tube is eluted with near average linear velocity, while the analyte largely partitioned in the outer phase is eluted with a smaller velocity than the average linear velocity. The elution times of the analytes can be easily reversed by changing the component ratio of solvents in the carrier solution.

\section{Analytical conditions}

The analyte-mixture solution must be injected into the capillary inlet of Capillary A, because the analyte zone in Capillary B is never formed exactly through the T-type joint if the analyte solution is injected into the inlet of Capillary B. Also, 2,6-naphthalenedisulfonic acid that was one of the analyte components was not enough dissolved with Mixture B (acetonitrile-ethyl acetate mixture), even not with acetonitrile alone, to show an absorption signal. Consequently, the analyte-mixture solution of 1-naphthol and 2,6-naphthalenedisulfonic acid dissolved with Mixture A (water-acetonitrile mixture) was injected into Capillary A and delivered toward Capillary B.

The Mixtures A and B were delivered at the various flow rates of 1.0 and 8.0, 2.0 and 6.0, 4.0 and 4.0, 6.0 and 2.0, as well as 8.0 and $1.0 \mu \mathrm{min}^{-1}$, respectively. The baselines monitored by the absorption detector were stable for the flow rates of 1.0 and 8.0, 4.0 and 4.0, as well as 8.0 and $1.0 \mu \mathrm{min}^{-1}$ for Mixtures A 
and B. But they were not stable for the flow rates of 2.0 and 6.0 or for 6.0 and $4.0 \mu 1 \mathrm{~min}^{-1}$. The carrier solution (water-acetonitrile-ethyl acetate mixture) provided by mixing Mixtures A and B at the end of Capillary A in Capillary B must be homogeneous for the stable baselines observed through absorption detection, while it must be heterogeneous for the unstable baselines.

Furthermore, as a preliminary experiment, when Mixtures A and $\mathrm{B}$ were fed at the flow rates of the respective 4.0 and $4.0 \mu 1 \mathrm{~min}^{-1}$ into the capillaries, the analytes in the mixture solution were not separated; the carrier solution obtained by the flow rates comprised the water-acetonitrile-ethyl acetate mixture with the volume ratio of 3:5:1. The volume ratio that did not possess a large unbalance in water-organic solvent ratio must not lead to separation in the present system, although it could provide the stable baseline. As a result, we adopted the flow rates of 1.0 and 8.0 as well as 8.0 and $1.0 \mu \mathrm{min}^{-1}$ for the Mixtures A and B in the following experiments.

\section{Effect of the temperature of Capillary $B$}

First, Capillary B having a 100-cm effective length for separation was used in the present TRDC system. The capillary length was very common in our previous works, ${ }^{9,10}$ but we had not used the capillary tube of $250 \mu \mathrm{m}$ i.d. (the largest inner diameter of the tube that we had used in the TRDC system was $200 \mu \mathrm{m}$ of polyethylene tube). ${ }^{9}$ When Capillary B having a 100 -cm effective length was used, the obtained chromatographic data lacked reproducibility, although the details are not described. Then, we tried to use Capillary B having a $200-\mathrm{cm}$ effective length and dipped one part of it $(c a .130 \mathrm{~cm})$ in water to control the capillary temperature. The data were obtained with good reproducibility at a capillary temperature of $15-20^{\circ} \mathrm{C}$. In a large inner diameter tube of $250 \mu \mathrm{m}$ i.d. that was necessitated for developing the double capillary tubes, the capillary temperature control must be useful for providing stable tube radial distribution of carrier solvents in the capillary tube. In addition the analytes were never separated at a capillary temperature of more than $25^{\circ} \mathrm{C}$. The following experiments were carried out using Capillary B of $200 \mathrm{~cm}$ effective length at the capillary temperature of $15-20^{\circ} \mathrm{C}$.

\section{Separation performance}

Mixture A (water-acetonitrile mixture) and Mixture B (acetonitrile-ethyl acetate mixture) were delivered into the large Capillary B from the inside and outside, respectively, at the flow rates of 1.0 and 8.0 as well as 8.0 and $1.0 \mu \mathrm{min}^{-1}$, and then mixed through the tube to provide a water-acetonitrile-ethyl acetate carrier solution. We could control the component ratio of carrier solvents in the carrier solution, that is, we could determine if the solution was organic solvent-rich or water-rich, by controlling the flow rates of the mixture solutions. The following component ratios of carrier solvents were demonstrated according to the flow rates mentioned above: organic solvent-rich (water-acetonitrile-ethyl acetate, volume ratio 3:33:8) and water-rich (water-acetonitrile-ethyl acetate, volume ratio 24:12:1).

A model analyte mixture (1-naphthol and 2,6-naphthalenedisulfonic acid) was subjected to the TRDC system that was adjusted to feed the organic solvent-rich carrier solution. The two components were eluted in the order listed under laminar flow conditions and detected by the absorption detector. Similarly, the model analyte mixture was subjected to the TRDC system that was adjusted to feed the water-rich carrier solution. The two components were eluted in the reverse order. Obtained chromatograms for these two experiments are shown in Fig. 2.
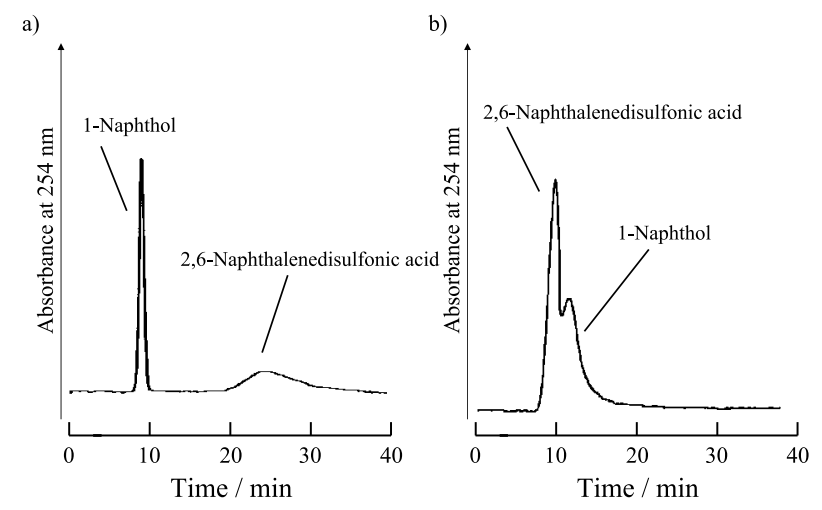

Fig. 2 Chromatograms of a mixture of 1-naphthol and 2,6-naphthalenedisulfonic acid obtained using the present TRDC system equipped with double capillary tubes: a) organic solvent-rich carrier solution (water-acetonitrile-ethyl acetate, volume ratio 3:33:8), and b) water-rich carrier solution (water-acetonitrile-ethyl acetate, volume ratio 24:12:1). Analyte concentrations are each $2 \mathrm{mM}$.

The components of the analytes, 1-naphthol and 2,6-naphthalenedisulfonic acid, in the chromatograms were confirmed with individual absorption signals. The elution times of the model analyte mixtures were easily changed by altering the component ratio of the carrier solvents in the carrier solution. We altered the component ratio by adjusting the flow rates of the mixture solutions in the double capillary tubes.

Additionally, instead of Mixture B of acetonitrile-ethyl acetate mixture, we attempted to use a solution containing acetonitrile alone that was fed into Capillary B. Such an analyte-mixture solution was never separated at any flow rates. Ethyl acetate (hydrophobic organic solvent) made an important role for separation performance in the TRDC system.

\section{Tube radial distribution of carrier solvents}

It should be emphasized that analyte separation was achieved with even the organic solvent-rich carrier solution. Even when the water-acetonitrile (water-rich) solution was fed from the inner tube and the acetonitrile-ethyl acetate (organic solvent-rich) solution from the outside through the T-type joint, the organic solvent-rich carrier solution that resulted upon their mixing through Capillary B was delivered to the detector, providing that the organic solvent-rich phase formed in the middle of the tube far from the inner wall (the inner phase) and the water-rich phase formed near the inner wall of the tube (the outer or capillary wall phase). That is, the data obtained here supported that tube radial distribution of carrier solvents in a carrier solution was appropriately performed under laminar flow conditions in the present TRDC system.

In conclusion, we have developed a TRDC system equipped with double capillary tubes having different inner diameters. We could change the component ratio of carrier solvents in the carrier solution by adjusting the flow rates of the two mixture solutions delivered into the tubes in the system, not preparing carrier solutions beforehand and exchanging them. A model analyte mixture solution of 1-naphthol and 2,6-naphthalenedisulfonic acid was eluted, in the order listed, with the organic solvent-rich carrier solution and eluted in the opposite order with the water-rich carrier solution. The demonstration shown here supported the specific solvent distribution in the capillary tube, i.e., the tube radial distribution of the carrier solvents, 
in the TRDC system together with the experimental data reported previously. ${ }^{9,10}$

\section{Acknowledgements}

This work was supported by a Grant-in-Aid for Scientific Research (C) from the Ministry of Education, Culture, Sports, Science, and Technology, Japan. It was also supported by the Academic Frontier Research Project on "New Frontiers of Biomedical Engineering Research" of the Ministry of Education, Culture, Sports, Science and Technology, Japan.

\section{References}

1. X. Wang, X. Lin, Z. Xie, and J. P. Giesy, J. Chromatogr., A,
2009, 1216, 4611.

2. I. Mikšik and P. Sedláková, J. Sep. Sci., 2007, 30, 1686.

3. M. C. Jung, N. Munro, G. Shi, A. C. Michael, and S. G. Weber, Anal. Chem., 2006, 78, 1761.

4. J. Urban and P. Jandera, J. Sep. Sci., 2008, 31, 2521.

5. K. Otsuka and S. Terabe, Trends Anal. Chem., 1993, 12, 125.

6. R. Koike, F. Kitagawa, and K. Otsuka, J. Sep. Sci., 2009, 32, 399.

7. M. Tabata, Y. G. Wu, T. Charoenraks, and S. S. Samaratunga, Bull. Chem. Soc. Jpn., 2006, 79, 1742.

8. T. Charoenraks, M. Tabata, and K. Fujii, Anal. Sci., 2008, $24,1239$.

9. N. Jinno, M. Hashimoto, and K. Tsukagoshi, Anal. Sci., 2009, 25, 145.

10. N. Jinno, M. Itano, M. Hashimoto, and K. Tsukagoshi, Talanta, 2009, 78, 1348. 